Article

\title{
Rapid Determination of $\beta$-Glucan Content of Hulled and Naked Oats Using near Infrared Spectroscopy Combined with Chemometrics
}

\author{
Maninder Meenu ${ }^{1,+}{ }^{+}$, Yaqian Zhang ${ }^{1,+}$, Uma Kamboj $^{2}$, Shifeng Zhao ${ }^{3}$, Lixia Cao ${ }^{3}$, Ping He ${ }^{4}$ and Baojun Xu ${ }^{1, *}$ (D) \\ 1 Food Science and Technology Program, BNU-HKBU United International College, Zhuhai 519087, China; \\ meenu_maninder@yahoo.com (M.M.); yaqianzhang_007@link.cuhk.edu.hk (Y.Z.) \\ 2 School of Chemical Engineering and Physical Sciences, Lovely Professional University, \\ Jalandhar 144411, India; amu_kam@yahoo.com \\ 3 Zhangjiakou Academy of Agricultural Sciences, Zhangjiakou 075000, China; zsf1886@163.com (S.Z.); \\ ymsclx@163.com (L.C.) \\ 4 Statistical Program, BNU-HKBU United International College, Zhuhai 519087, China; heping@uic.edu.cn \\ * Correspondence: baojunxu@uic.edu.cn; Tel.: +86-756-3620636 \\ + These authors contributed equally to this work.
}

check for

updates

Citation: Meenu, M.; Zhang, Y.;

Kamboj, U.; Zhao, S.; Cao, L.; He, P.; $\mathrm{Xu}, \mathrm{B}$. Rapid Determination of $\beta$-Glucan Content of Hulled and Naked Oats Using near Infrared Spectroscopy Combined with Chemometrics. Foods 2022, 11, 43 . https://doi.org/10.3390/ foods11010043

Academic Editor: Stefania Cesa

Received: 20 November 2021

Accepted: 21 December 2021

Published: 24 December 2021

Publisher's Note: MDPI stays neutral with regard to jurisdictional claims in published maps and institutional affiliations.

Copyright: (C) 2021 by the authors. Licensee MDPI, Basel, Switzerland. This article is an open access article distributed under the terms and conditions of the Creative Commons Attribution (CC BY) license (https:// creativecommons.org/licenses/by/ $4.0 /)$.

\begin{abstract}
The quantification of $\beta$-glucan in oats is of immense importance for plant breeders and food scientists to develop plant varieties and food products with a high quantity of $\beta$-glucan. However, the chemical analysis of $\beta$-glucan is time consuming, destructive, and laborious. In this study, near-infrared (NIR) spectroscopy in conjunction with Chemometrics was employed for rapid and non-destructive prediction of $\beta$-glucan content in oats. The interval Partial Least Square (iPLS) along with correlation matrix plots were employed to analyze the NIR spectrum from 700-1300 nm, 1300-1900 nm, and 1900-2500 nm for the selection of important wavelengths for the prediction of $\beta$ glucan. The NIR spectral data were pre-treated using Savitzky Golay smoothening and normalization before employing partial least square regression (PLSR) analysis. The PLSR models were established based on the selection of wavelengths from PLS loading plots that present a high correlation with $\beta$ glucan content. It was observed that wavelength region $700-1300 \mathrm{~nm}$ is sufficient for the satisfactory prediction of $\beta$-glucan of hulled and naked oats with $\mathrm{R}^{2} \mathrm{c}$ of 0.789 and 0.677 , respectively, and RMSE $<0.229$.
\end{abstract}

Keywords: NIR spectroscopy; $\beta$-glucan; oats; prediction; partial least square regression

\section{Introduction}

Oats are produced in various countries around the world. According to the Food and Agriculture Organisation of United Nations, Russia is the top producer of oats (4.72 million metric tons $(\mathrm{mmt}))$ followed by Canada $(3.44 \mathrm{mmt})$, Spain $(1.48 \mathrm{mmt})$, Australia $(1.23 \mathrm{mmt})$, Poland $(1.17 \mathrm{mmt})$, and China $(1.00 \mathrm{mmt})$ [1]. There are about seventy species of oats around the world, while hulled oats (Avena sativa L.) and naked oats (A. nuda L.) are the most cultivated oats species. The latter is hull-free and exhibits a higher energy level compared with hulled oats [2]. Oats are a rich source of soluble fibers, mainly, $\beta$-glucan [3]. Primarily, $\beta$-glucan is present in the cell walls of the aleurone layer and starchy endosperm of oat grains [4]. It is a homopolysaccharide of long linear chains of glucose units connected by $\beta-(1 \rightarrow 3)$ and $\beta-(1 \rightarrow 4)$ linkages $[5,6]$.

$\beta$-glucan is also known as a functional and bioactive food ingredient due to its potential health benefits such as antitumor, anti-inflammatory, antioxidant, hypoglycemic, hypocholesterolemic, and immunomodulatory activities $[3,5]$. Thus, the demand for food products with a high level of $\beta$-glucan has been increased worldwide. Oats are used as human food in the form of cereals, breads, beverages, and infant foods [3]. $\beta$-glucans are also employed in the pharmaceutical industry to produce wound dressing material; 
for the treatment of burn injuries, skin substitute, and bone-substituting material; and in arthritis treatment. Moreover, $\beta$-glucans are also used in the cosmetics industry due to their anti-aging, moisturizing, and wound healing effects [5,7]. However, high $\beta$-glucan content is undesirable in the case of monogastric animal feed due to the digestion problem and reduced nutritional value of the feed [8].

To develop a new plant variety with a high level of $\beta$-glucan, the quantification of $\beta$ glucan is also important for plant breeders for the selection of high-quality oat cultivar. The McCleary enzymatic method is the most widely used method for the determination of $\beta$ glucan [9]. The primary step of this method involves disintegration and homogenization of oat grains that may induce variations in the $\beta$-glucan measurement if they are not properly conducted. Moreover, this chemical method is destructive, requires a long determination time, and is costly. During plant breeding experiments, extensive chemical analysis has to be conducted to explore the target genotype. Thus, there is an immediate requirement to substitute conventional analysis with up-to-date non-destructive, chemical-free, rapid, and easy techniques.

Near-infrared spectroscopy (NIRS) meets the requirements of plant breeders and food scientists to detect the $\beta$-glucan of oats without chemicals, in less time, and with minimum sample preparation and cost. NIR spectrum ranges from 700 to $2500 \mathrm{~nm}$ consist of broad overtone and combination bands of certain fundamental vibrations of $\mathrm{C}-\mathrm{H}$, $\mathrm{N}-\mathrm{H}$, and $\mathrm{O}-\mathrm{H}$ bonds present in organic compounds of grain samples [10]. NIRS in conjunction with chemometrics has been successfully employed in medical science [11], pharmaceuticals [12], and food industries [10,13,14]. In recent decades, NIRS along with modified partial least square regression was used for the accurate prediction (coefficient of determination for calibration $\left(\mathrm{R}^{2} \mathrm{C}\right)$ of 0.80 and coefficient of determination for prediction $\left(\mathrm{R}^{2} \mathrm{p}\right)$ of 0.75$)$ of groat percentage in oats from Italy [13]; neutral detergent fiber, acid detergent fiber, and acid detergent lignin of oats collected from Italy $\left(R^{2} \mathrm{C}=0.92-0.95\right.$ and coefficient of determination for cross-validation $\left.\left(R^{2} \mathrm{cv}\right)=0.83-0.93\right)$ [14]; nitrogen $\left(R^{2} \mathrm{C}=0.973\right.$, coefficient of determination for validation $\left.\left(R^{2} v\right)=0.984\right)$ and protein content $\left(R^{2} \mathrm{c}=0.973,0.965, \mathrm{R}^{2} \mathrm{v}=0.984,0.973\right)$ of food products containing oats [15], protein, and lipid contents of oats from Brazil [16]; and the detection of oat flour adulteration with wheat flour [17]. Previously, NIRS in the region 1194-1240 nm was also successfully employed to predict the accumulation of $(1 \rightarrow 3)(1 \rightarrow 4)$ - $\beta$-D-glucan while grain filling in barley [18]. In addition, NIRS was also employed to predict the $\beta$-glucan content of different oats genotypes collected from Italy and other European countries [4,19]. Previously, different types of NIR Instruments were explored for their suitability in measuring $\beta$-glucan content in naked barley. The Fourier transform near-infrared (FT-NIR) reflection instrument was reported to predict $\beta$-glucan with high efficiency $\left(R^{2} c=0.96-0.98\right)$ [20]. Furthermore, the NIR spectrum of 288 seeds of whole oat groats for six varieties grown in the USA was collected and used for the prediction of $\beta$-glucan content by employing Partial Least Square regression (PLSR) analysis [21]. Recently, NIR spectroscopy in conjunction with chemometrics was also employed for the efficient prediction of $\beta$-glucan content of hulled oats samples collected from diverse geographical regions of the USA [22].

However, to the best of our knowledge, no study has been conducted for the prediction of $\beta$-glucan content of hulled and naked oats collected from China. Thus, this study was carried out to predict the $\beta$-glucan content of oats from China, by using NIRS in conjunction with PLSR analysis. In this study, we also included certain oat samples collected from the United States and Canada to increase the genetic and geographical variability of the sample pool.

\section{Materials and Methods}

\subsection{Collection of Oat Samples}

A total of 179 different varieties of oat samples (100 naked and 79 hulled oats) were procured from Zhangjiakou Agricultural Academy, Hebei Province, China. The samples were cleaned manually to remove unwanted materials and damaged grains and then refrig- 
erated at $\left(2-6{ }^{\circ} \mathrm{C}\right)$ until further analysis. The samples were brought at room temperature before chemical and spectral analysis followed by dehulling and removal of impurities. The final oat groats were finely ground by a regular laboratory mill (400A, Yongkang City Zhanfan Industry and Trade Co., Ltd., Yongkang, Zhejiang, China) and passed through a 60 mesh screen $(\mathrm{d}=0.25 \mathrm{~mm})$ to achieve homogeneous particle size that, in turn, reduce the problem of scattering during NIR data acquisition. The resultant oat flours were further used for chemical and spectral analysis.

\subsection{Chemicals}

The analytical grade sodium dihydrogen orthophosphate dihydrate $\left(\mathrm{NaH}_{2} \mathrm{PO}_{4} \cdot 2 \mathrm{H}_{2} \mathrm{O}\right)$, sodium hydroxide $(\mathrm{NaOH})$, and glacial acetic acid were purchased from Tianjin Damao Chemical Reagent Co., Ltd. (Tianjin, China). The $\beta$-glucan content of oat samples (reference data) was determined by using the mixed-linkage beta-glucan kit (K-BGLU, Megazyme, Bray, Ireland).

\subsection{Determination of $\beta$-Glucan Content}

The mixed-linkage $\beta$-glucan assay procedure (McCleary method) was used to determine the $\beta$-glucan content of oat samples [9]. Briefly, the $120 \mathrm{mg}$ of oat sample was suspended in $0.2 \mathrm{~mL}$ of $50 \%$ ethanol and hydrated in a sodium phosphate-buffered solution (4 mL, $20 \mathrm{mM}, \mathrm{pH}$ 6.5). Furthermore, the reaction mixture was incubated with purified lichenase enzyme $(0.2 \mathrm{~mL})$ and filtered. An aliquot of the filtrate was then hydrolyzed with purified $\beta$-glucosidase $(0.1 \mathrm{~mL}$ in $50 \mathrm{mM}$ sodium acetate buffer $\mathrm{pH} 4.0)$ to release D-glucose followed by the addition of glucose oxidase/peroxidase reagent $(3.0 \mathrm{~mL})$ and incubation for $20 \mathrm{~min}$ at $50{ }^{\circ} \mathrm{C}$. The absorbance of the solution was measured at $510 \mathrm{~nm}$ within $1 \mathrm{~h}$. The $\beta$-glucan content of each sample was analyzed in triplicates.

\subsection{Acquisition of NIR Spectra}

The NIR spectrum of the oat flour sample was measured by using Frontier FT-IR/NIR Spectrometer (PerkinElmer Inc., Waltham, MA, USA) using a NIR infrared reflectance accessory-NIRA (PerkinElmer Inc., Waltham, MA, USA) equipped with a spinning sample module and Spectrum-V.7.3.1.1023 (PerkinElmer Inc., Waltham, MA, USA) software. The spectrum was recorded in diffuse reflectance mode from 700 to $2500 \mathrm{~nm}$ using 32 scans at $1 \mathrm{~nm}$ intervals. The measurements were performed in duplicate, and the average spectrum was used for further analysis.

\subsection{Statistical Analysis}

The interaction of NIR radiation with oat samples gives rise to a complex spectrum. This spectrum comprises overtone vibrations and combination bands at particular wavelengths due to the bonds present in organic compounds of oats. Thus, for the extraction of useful information from spectra and quantitative analysis of $\beta$-glucan present in samples, multivariate statistical analysis was carried out by employing reference data of $\beta$-glucan as primary data and spectral data as secondary data. The multivariate analysis was performed by using chemometric software (The Unscrambler X version 10.3; CAMO Software AS, Oslo, Norway). Random sampling was conducted to divide oats samples into two categories-calibration and prediction set with the ratio of 4:1. In case of hulled oats, 64 samples were included in the calibration set, whereas 15 samples were kept in the prediction set. However, in the case of naked oats, 80 samples were kept in the calibration set and 20 samples were used to develop the prediction model [10]. The spectral data were maximum normalized, and a baseline correction was performed for the obtained spectra. Savitzky-Golay smoothening was applied to the spectral data. The complete NIR wavelength range was divided into three equal parts and equal width (for both naked and hulled oat samples) namely, 700-1300 nm (I), 1300-1900 nm (II), and 1900-2500 nm (III) followed by developing a correlation matrix for each section of wavelength with the $\beta$-glucan data of oat samples obtained by using reference analysis. PLSR and interval PLS 
regression (iPLS) was applied using Nonlinear Iterative Partial Least Squares (NIPALS) algorithm, and PCA analysis was performed on the spectral data as a whole and on the three parts each. Regression models were developed for each part for the determination of the $\beta$-glucan content in the samples.

\section{Results}

The McCleary method was used as a reference method for the determination of $\beta$ glucan content in seventy-nine oats varieties. The $\beta$-glucan content and moisture content of hulled and naked oat varieties under investigation are mentioned in Tables 1 and 2. The moisture content of hulled and naked oat samples varied from $4.86 \%$ to $6.43 \%$, and $4.17 \%$ to $6.19 \%$. In the case of hulled oats, the highest amount of $\beta$-glucan (5.5\%) was observed in ZNY 258 followed by $5.25 \%$ in the case of zyp2 gp 63-56, and $4.94 \%$ in the case of zyp2 gp 86-79, whereas oat variety Zhangyan \#7 presented the lowest amount of $\beta$-glucan (3.1\%). In the case of naked oats, the highest amount of $\beta$-glucan (5.22\%) was found in sample BY 4 , followed by $5.21 \%$ in Bayan \#9 and by $5.17 \%$ in BY 34 . However, the lowest content (3.12\%) was observed in the case of sample BY 37.

Table 1. $\beta$-glucan content, moisture content, and origin of hulled oat samples.

\begin{tabular}{|c|c|c|c|c|}
\hline S. No. & Sample Name & Moisture Content (\%) & $\begin{array}{c}\beta \text {-Glucan } \% w / w \\
\text { (Dry wt. Basis) }\end{array}$ & Origin \\
\hline 1 & zyp2 gp 016-14 & $5.91 \pm 0.04$ & $4.74 \pm 0.06$ & Zhangjiakou, Hebei, China \\
\hline 2 & zyp2 gp 002-1 & $5.65 \pm 0.04$ & $4.14 \pm 0.04$ & Zhangjiakou, Hebei, China \\
\hline 3 & zyp2 gp 035-30 & $5.64 \pm 0.04$ & $4.65 \pm 0.12$ & Zhangjiakou, Hebei, China \\
\hline 4 & zyp2 gp 129-119 & $5.68 \pm 0.04$ & $4.35 \pm 0.19$ & Zhangjiakou, Hebei, China \\
\hline 5 & zyp2 gp 135-65 & $6.41 \pm 0.11$ & $4.77 \pm 0.10$ & Zhangjiakou, Hebei, China \\
\hline 6 & zyp2 gp -74 & $5.99 \pm 0.67$ & $4.63 \pm 0.03$ & Zhangjiakou, Hebei, China \\
\hline 7 & zyp2 gp 023-20 & $6.30 \pm 0.67$ & $4.77 \pm 0.13$ & Zhangjiakou, Hebei, China \\
\hline 8 & zyp2 gp 78-71 & $5.70 \pm 0.04$ & $4.35 \pm 0.01$ & Zhangjiakou, Hebei, China \\
\hline 9 & zyp2 gp 74-67 & $5.42 \pm 0.04$ & $4.56 \pm 0.03$ & Zhangjiakou, Hebei, China \\
\hline 10 & zyp2 gp 015-13 & $5.66 \pm 0.42$ & $4.59 \pm 0.05$ & Zhangjiakou, Hebei, China \\
\hline 11 & zyp2 gp 107-100 & $5.32 \pm 0.62$ & $4.01 \pm 0.11$ & Zhangjiakou, Hebei, China \\
\hline 12 & zyp2 gp 027-24 & $5.33 \pm 0.48$ & $4.33 \pm 0.03$ & Zhangjiakou, Hebei, China \\
\hline 13 & zyp2 gp 137-07 & $5.67 \pm 0.56$ & $3.82 \pm 0.03$ & Zhangjiakou, Hebei, China \\
\hline 14 & zyp2 gp 70-63 & $5.19 \pm 0.16$ & $4.68 \pm 0.04$ & Zhangjiakou, Hebei, China \\
\hline 15 & zyp2 gp 76-69 & $5.18 \pm 0.08$ & $3.59 \pm 0.06$ & Zhangjiakou, Hebei, China \\
\hline 16 & zyp2 gp 017-15 & $5.48 \pm 0.50$ & $4.61 \pm 0.14$ & Zhangjiakou, Hebei, China \\
\hline 17 & zyp2 gp 61-54 & $5.64 \pm 0.09$ & $4.61 \pm 0.12$ & Zhangjiakou, Hebei, China \\
\hline 18 & zyp2 gp 031-27 & $5.47 \pm 0.52$ & $4.71 \pm 0.00$ & Zhangjiakou, Hebei, China \\
\hline 19 & zyp2 gp 104-97 & $5.20 \pm 0.37$ & $4.4 \pm 0.04$ & Zhangjiakou, Hebei, China \\
\hline 20 & zyp2 gp 91-84 & $5.50 \pm 0.57$ & $4.51 \pm 0.03$ & Zhangjiakou, Hebei, China \\
\hline 21 & zyp2 gp 82-75 & $6.14 \pm 0.26$ & $4.34 \pm 0.00$ & Zhangjiakou, Hebei, China \\
\hline 22 & zyp2 gp 63-56 & $5.70 \pm 0.57$ & $5.25 \pm 0.03$ & Zhangjiakou, Hebei, China \\
\hline 23 & zyp2 gp 57-51 & $6.43 \pm 0.43$ & $4.87 \pm 0.05$ & Zhangjiakou, Hebei, China \\
\hline 24 & zyp2 gp 86-79 & $5.04 \pm 0.45$ & $4.94 \pm 0.08$ & Zhangjiakou, Hebei, China \\
\hline 25 & $\begin{array}{c}\text { zyp2 gp 20B PSX } \\
\text { 17G-26 }\end{array}$ & $5.42 \pm 0.42$ & $3.92 \pm 0.04$ & Zhangjiakou, Hebei, China \\
\hline
\end{tabular}


Table 1. Cont.

\begin{tabular}{|c|c|c|c|c|}
\hline S. No. & Sample Name & Moisture Content (\%) & $\begin{array}{c}\beta \text {-Glucan } \% w / w \\
\text { (Dry wt. Basis) }\end{array}$ & Origin \\
\hline 26 & zyp2 gp 083-2 & $6.18 \pm 0.39$ & $3.97 \pm 0.14$ & Zhangjiakou, Hebei, China \\
\hline 27 & zyp2 gp 024-21 & $5.84 \pm 0.40$ & $3.55 \pm 0.02$ & Zhangjiakou, Hebei, China \\
\hline 28 & IYP gp-6 & $5.58 \pm 0.57$ & $4.4 \pm 0.02$ & Zhangjiakou, Hebei, China \\
\hline 29 & zyp2 gp 67-60 & $5.69 \pm 0.21$ & $4.02 \pm 0.11$ & Zhangjiakou, Hebei, China \\
\hline 30 & Zhangyan \#2 & $5.76 \pm 0.12$ & $4.1 \pm 0.08$ & Zhangjiakou, Hebei, China \\
\hline 31 & Yibaiyan \#1 & $5.65 \pm 0.47$ & $4.49 \pm 0.04$ & United States \\
\hline 32 & Qinghai 444 & $5.62 \pm 0.06$ & $4.32 \pm 0.01$ & Xining, Qinghai, China \\
\hline 33 & Qingyin \#1 & $5.45 \pm 0.08$ & $3.98 \pm 0.08$ & Xining, Qinghai, China \\
\hline 34 & Qingyin \#1 & $5.51 \pm 0.32$ & $3.6 \pm 0.00$ & Xining, Qinghai, China \\
\hline 35 & Kanyan \#1 & $5.69 \pm 0.40$ & $3.8 \pm 0.04$ & \\
\hline 36 & Yizhangyan \#4 & $5.31 \pm 0.19$ & $3.64 \pm 0.02$ & Zhangjiakou, Hebei, China \\
\hline 37 & Dingyan \#2 & $5.60 \pm 0.01$ & $4.32 \pm 0.00$ & Dingxi, Gansu, China \\
\hline 38 & Jingbaiyan \#2 & $5.55 \pm 0.06$ & $3.69 \pm 0.10$ & \\
\hline 39 & Anrui & $5.7 \pm 0.13$ & $4.28 \pm 0.07$ & United States \\
\hline 40 & Qinghai sweet oat & $5.61 \pm 0.08$ & $4.12 \pm 0.03$ & Xining, Qinghai, China \\
\hline 41 & Kanyan \#5 & $6.19 \pm 0.52$ & $3.78 \pm 0.13$ & \\
\hline 42 & Zhangyan \#7 & $5.67 \pm 0.08$ & $3.1 \pm 0.06$ & Zhangjiakou, Hebei, China \\
\hline 43 & Qingyan \#1 & $5.50 \pm 0.11$ & $4.69 \pm 0.00$ & Xining, Qinghai, China \\
\hline 44 & Baiyan \#7 & $5.52 \pm 0.18$ & $4.38 \pm 0.01$ & \\
\hline 45 & Linna & $5.57 \pm 0.40$ & $4.31 \pm 0.04$ & Canada \\
\hline 46 & Kanyan \#4 & $5.47 \pm 0.42$ & $3.57 \pm 0.03$ & \\
\hline 47 & Baiyan \#14 & $5.35 \pm 0.42$ & $3.48 \pm 0.19$ & \\
\hline 48 & Kanyan \#2 & $5.19 \pm 0.10$ & $3.6 \pm 0.08$ & \\
\hline 49 & Zhangyan \#3 & $5.21 \pm 0.12$ & $4.47 \pm 0.06$ & Zhangjiakou, Hebei, China \\
\hline 50 & Dingyin \#1 & $5.15 \pm 0.22$ & $3.81 \pm 0.02$ & Dingxi, Gansu, China \\
\hline 51 & Kanyan \#3 & $5.36 \pm 0.08$ & $4.13 \pm 0.01$ & \\
\hline 52 & Mingcui & $5.60 \pm 0.11$ & $4.25 \pm 0.10$ & United States \\
\hline 53 & Yibaiyan \#3 & $4.87 \pm 0.59$ & $4.67 \pm 0.10$ & United States \\
\hline 54 & Zhangyan \#1 & $5.87 \pm 0.52$ & $4.03 \pm 0.01$ & Zhangjiakou, Hebei, China \\
\hline 55 & Kanyan \#6 & $5.11 \pm 0.40$ & $4.08 \pm 0.03$ & \\
\hline 56 & Lezhen & $5.53 \pm 0.45$ & $4.35 \pm 0.00$ & United States \\
\hline 57 & Zhangyan \#4 & $5.96 \pm 0.30$ & $4.74 \pm 0.01$ & Zhangjiakou, Hebei, China \\
\hline 58 & Beile & $5.50 \pm 0.45$ & $3.91 \pm 0.00$ & Canada \\
\hline 59 & Jiayan \#2 & $5.37 \pm 0.04$ & $3.79 \pm 0.11$ & Canada \\
\hline 60 & ZNY 221 & $5.57 \pm 0.06$ & $3.97 \pm 0.02$ & Zhangjiakou, Hebei, China \\
\hline 61 & ZNY 202 & $6.17 \pm 0.33$ & $4.32 \pm 0.04$ & Zhangjiakou, Hebei, China \\
\hline 62 & ZNY 300 & $5.48 \pm 0.46$ & $4.14 \pm 0.00$ & Zhangjiakou, Hebei, China \\
\hline
\end{tabular}


Table 1. Cont.

\begin{tabular}{ccccc}
\hline S. No. & Sample Name & Moisture Content (\%) & $\begin{array}{c}\beta \text {-Glucan } \% \text { w/w } \\
\text { (Dry wt. Basis) }\end{array}$ & Origin \\
\hline 63 & ZNY 303 & $5.61 \pm 0.06$ & $3.82 \pm 0.04$ & Zhangjiakou, Hebei, China \\
\hline 64 & ZNY 297 & $5.65 \pm 0.43$ & $4.48 \pm 0.02$ & Zhangjiakou, Hebei, China \\
\hline 65 & ZNY 218 & $5.54 \pm 0.44$ & $4.17 \pm 0.00$ & Zhangjiakou, Hebei, China \\
\hline 66 & ZNY 293 & $6.15 \pm 0.47$ & $4.78 \pm 0.06$ & Zhangjiakou, Hebei, China \\
\hline 67 & ZNY 225 & $5.57 \pm 0.60$ & $4.38 \pm 0.00$ & Zhangjiakou, Hebei, China \\
\hline 68 & ZNY 254 & $5.58 \pm 0.66$ & $3.27 \pm 0.00$ & Zhangjiakou, Hebei, China \\
\hline 69 & ZNY 233 & $5.19 \pm 0.08$ & $4.62 \pm 0.04$ & Zhangjiakou, Hebei, China \\
\hline 70 & ZNY 290 & $5.33 \pm 0.23$ & $3.7 \pm 0.03$ & Zhangjiakou, Hebei, China \\
\hline 71 & ZNY 205 & $5.78 \pm 0.38$ & $4.48 \pm 0.17$ & Zhangjiakou, Hebei, China \\
\hline 72 & ZNY 232 & $5.36 \pm 0.28$ & $4.13 \pm 0.04$ & Zhangjiakou, Hebei, China \\
\hline 73 & ZNY 248 & $5.40 \pm 0.30$ & $4.44 \pm 0.07$ & Zhangjiakou, Hebei, China \\
\hline 74 & ZNY 288 & $5.41 \pm 0.34$ & $3.23 \pm 0.02$ & Zhangjiakou, Hebei, China \\
\hline 75 & ZNY 231 & $5.44 \pm 0.39$ & $4.51 \pm 0.03$ & Zhangjiakou, Hebei, China \\
\hline 76 & ZNY 251 & $5.68 \pm 0.72$ & $5.5 \pm 0.08$ & Zhangjiakou, Hebei, China \\
\hline 77 & ZNY 258 & $5.62 \pm 0.15$ & $4.83 \pm 0.06$ & Zhangjiakou, Hebei, China \\
\hline 78 & ZNY 255 & $5.77 \pm 0.60$ & $4.01 \pm 0.01$ & Zhangjiakou, Hebei, China \\
\hline 79 & ZNY239 & $4.84 \pm 0.31$ & Zhangjiakou, Hebei, China \\
\hline
\end{tabular}

Data were expressed as mean \pm standard deviation $(n=3), w / w$; weight/weight.

Table 2. $\beta$-glucan content, moisture content, and origin of naked oat samples.

\begin{tabular}{|c|c|c|c|c|}
\hline S. No. & Sample Name & Moisture Content (\%) & $\begin{array}{c}\beta \text {-Glucan } \% w / w \\
\text { (Dry wt. Basis) }\end{array}$ & Origin \\
\hline 1 & Yanke \#2 & $5.56 \pm 0.31$ & $3.96 \pm 0.06$ & Huhehaote, Inner Mongolia, China \\
\hline 2 & Baiyan \#2 & $5.84 \pm 0.39$ & $4.00 \pm 0.16$ & Baicheng, Jilin, China \\
\hline 3 & Kanyou \#6 & $5.74 \pm 0.14$ & $3.97 \pm 0.09$ & \\
\hline 4 & Dingyou \#9 & $5.71 \pm 0.29$ & $3.59 \pm 0.17$ & Dingxi, Gansu, China \\
\hline 5 & Baiyan \#11 & $5.63 \pm 0.49$ & $4.23 \pm 0.03$ & Baicheng, Jilin, China \\
\hline 6 & Kanyou \#18 & $5.76 \pm 0.05$ & $5.06 \pm 0.13$ & \\
\hline 7 & Jinyan \#8 & $5.97 \pm 0.23$ & $4.14 \pm 0.10$ & Datong, Shanxi, China \\
\hline 8 & Yuanza \#1 & $5.14 \pm 0.44$ & $3.74 \pm 0.05$ & Zhangjiakou, Hebei, China \\
\hline 9 & Caoyou \#1 & $5.91 \pm 0.66$ & $3.75 \pm 0.11$ & Huhehaote, Inner Mongolia, China \\
\hline 10 & Yanxuan 2007 & $5.61 \pm 0.07$ & $4.34 \pm 0.01$ & Datong, Shanxi, China \\
\hline 11 & Jinyan \#1 & $5.64 \pm 0.39$ & $3.82 \pm 0.03$ & Datong, Shanxi, China \\
\hline 12 & Bayan \#9 & $5.79 \pm 0.13$ & $5.21 \pm 0.08$ & \\
\hline 13 & Yizhangyou \#6 & $5.60 \pm 0.32$ & $4.28 \pm 0.08$ & Zhangjiakou, Hebei, China \\
\hline 14 & Baiyan \#15 & $5.55 \pm 0.43$ & $3.63 \pm 0.05$ & \\
\hline 15 & Baiyan \#8 & $5.49 \pm 0.68$ & $3.82 \pm 0.00$ & \\
\hline 16 & Zhangyou \#7 & $5.72 \pm 0.66$ & $3.93 \pm 0.12$ & \\
\hline
\end{tabular}


Table 2. Cont.

\begin{tabular}{|c|c|c|c|c|}
\hline S. No. & Sample Name & Moisture Content (\%) & $\begin{array}{c}\beta \text {-Glucan } \% w / w \\
\text { (Dry wt. Basis) }\end{array}$ & Origin \\
\hline 17 & Yizhangyou \#3 & $5.82 \pm 0.40$ & $4.44 \pm 0.01$ & Zhangjiakou, Hebei, China \\
\hline 18 & Yizhangyou \#12 & $5.52 \pm 0.38$ & $4.01 \pm 0.04$ & \\
\hline 19 & Yizhangyou \#2 & $6.18 \pm 0.57$ & $3.84 \pm 0.03$ & Zhangjiakou, Hebei, China \\
\hline 20 & Jinyinyan \#1 & $5.45 \pm 0.39$ & $4.54 \pm 0.03$ & Datong, Shanxi, China \\
\hline 21 & w85 & $5.44 \pm 0.10$ & $3.59 \pm 0.01$ & Baicheng, Jilin, China \\
\hline 22 & Yizhangyou \#5 & $5.58 \pm 0.42$ & $4.25 \pm 0.01$ & Zhangjiakou, Hebei, China \\
\hline 23 & Kanyou \#8 & $5.54 \pm 0.43$ & $4.23 \pm 0.04$ & \\
\hline 24 & Kanyou \#13 & $5.66 \pm 0.13$ & $3.80 \pm 0.02$ & \\
\hline 25 & Huazao \#2 & $5.51 \pm 0.47$ & $3.27 \pm 0.01$ & Zhangjiakou, Hebei, China \\
\hline 26 & Baiyan \#13 & $5.77 \pm 0.85$ & $4.22 \pm 0.01$ & \\
\hline 27 & Kanyou \#10 & $5.44 \pm 0.54$ & $4.14 \pm 0.09$ & \\
\hline 28 & Kanyou \#5 & $5.22 \pm 0.05$ & $4.50 \pm 0.02$ & \\
\hline 29 & Neiyan \#5 & $6.19 \pm 0.42$ & $3.98 \pm 0.07$ & \\
\hline 30 & Jinyan \#14 & $5.33 \pm 0.28$ & $4.05 \pm 0.10$ & Datong, Shanxi, China \\
\hline 31 & Kanyou \#3 & $5.43 \pm 0.42$ & $4.33 \pm 0.04$ & \\
\hline 32 & Ningyou \#1 & $5.94 \pm 0.61$ & $4.18 \pm 0.03$ & Guyuan, Ningxia, China \\
\hline 33 & Yizhangyou \#4 & $5.58 \pm 0.07$ & $3.73 \pm 0.03$ & Zhangjiakou, Hebei, China \\
\hline 34 & Yan 2009 & $5.39 \pm 0.08$ & $4.01 \pm 0.01$ & Datong, Shanxi, China \\
\hline 35 & Jinyan \#13 & $5.13 \pm 0.05$ & $4.06 \pm 0.06$ & Datong, Shanxi, China \\
\hline 36 & Yizhangyou \#5 & $5.31 \pm 0.11$ & $3.91 \pm 0.03$ & Zhangjiakou, Hebei, China \\
\hline 37 & Huawan \#6 & $5.50 \pm 0.56$ & $3.78 \pm 0.03$ & Zhangjiakou, Hebei, China \\
\hline 38 & Yuanza \#2 & $5.46 \pm 0.04$ & $4.43 \pm 0.06$ & Zhangjiakou, Hebei, China \\
\hline 39 & Ding you \#1 & $5.46 \pm 0.52$ & $3.82 \pm 0.00$ & Dingxi, Gansu, China \\
\hline 40 & Jinyan \#9 & $5.70 \pm 0.87$ & $3.13 \pm 0.00$ & Datong, Shanxi, China \\
\hline 41 & ZNY 242 & $5.83 \pm 0.83$ & $3.80 \pm 0.06$ & Zhangjiakou, Hebei, China \\
\hline 42 & ZNY 283 & $5.72 \pm 0.72$ & $3.55 \pm 0.00$ & Zhangjiakou, Hebei, China \\
\hline 43 & ZNY 273 & $5.17 \pm 0.52$ & $4.23 \pm 0.04$ & Zhangjiakou, Hebei, China \\
\hline 44 & ZNY 266 & $5.24 \pm 0.08$ & $4.31 \pm 0.02$ & Zhangjiakou, Hebei, China \\
\hline 45 & ZNY 272 & $5.81 \pm 0.74$ & $3.89 \pm 0.02$ & Zhangjiakou, Hebei, China \\
\hline 46 & ZNY 209 & $6.05 \pm 0.57$ & $3.84 \pm 0.10$ & Zhangjiakou, Hebei, China \\
\hline 47 & BY 1 & $5.59 \pm 0.32$ & $4.44 \pm 0.09$ & Zhangjiakou, Hebei, China \\
\hline 48 & BY 2 & $5.25 \pm 0.39$ & $4.98 \pm 0.17$ & Zhangjiakou, Hebei, China \\
\hline 49 & BY 3 & $5.37 \pm 0.66$ & $4.92 \pm 0.07$ & Zhangjiakou, Hebei, China \\
\hline 50 & BY 4 & $5.51 \pm 0.49$ & $5.22 \pm 0.03$ & Zhangjiakou, Hebei, China \\
\hline 51 & BY 5 & $5.32 \pm 0.79$ & $4.50 \pm 0.04$ & Zhangjiakou, Hebei, China \\
\hline 52 & BY 6 & $5.80 \pm 0.49$ & $4.45 \pm 0.03$ & Zhangjiakou, Hebei, China \\
\hline 53 & BY 7 & $5.75 \pm 0.51$ & $4.04 \pm 0.10$ & Zhangjiakou, Hebei, China \\
\hline 54 & BY 8 & $5.47 \pm 0.49$ & $4.28 \pm 0.09$ & Zhangjiakou, Hebei, China \\
\hline
\end{tabular}


Table 2. Cont.

\begin{tabular}{|c|c|c|c|c|}
\hline S. No. & Sample Name & Moisture Content (\%) & $\begin{array}{c}\beta \text {-Glucan } \% w / w \\
\text { (Dry wt. Basis) }\end{array}$ & Origin \\
\hline 55 & BY 9 & $6.03 \pm 0.28$ & $3.91 \pm 0.11$ & Zhangjiakou, Hebei, China \\
\hline 56 & BY 10 & $5.79 \pm 0.42$ & $4.31 \pm 0.02$ & Zhangjiakou, Hebei, China \\
\hline 57 & BY 11 & $5.62 \pm 0.62$ & $4.69 \pm 0.05$ & Zhangjiakou, Hebei, China \\
\hline 58 & BY 12 & $5.58 \pm 0.58$ & $4.19 \pm 0.05$ & Zhangjiakou, Hebei, China \\
\hline 59 & BY 13 & $5.49 \pm 0.42$ & $4.60 \pm 0.09$ & Zhangjiakou, Hebei, China \\
\hline 60 & BY 14 & $5.07 \pm 0.19$ & $4.52 \pm 0.09$ & Zhangjiakou, Hebei, China \\
\hline 61 & BY 15 & $5.54 \pm 0.49$ & $4.26 \pm 0.04$ & Zhangjiakou, Hebei, China \\
\hline 62 & BY 16 & $5.42 \pm 0.79$ & $4.47 \pm 0.00$ & Zhangjiakou, Hebei, China \\
\hline 63 & BY 17 & $5.13 \pm 0.49$ & $5.16 \pm 0.00$ & Zhangjiakou, Hebei, China \\
\hline 64 & BY 18 & $4.82 \pm 0.51$ & $4.44 \pm 0.02$ & Zhangjiakou, Hebei, China \\
\hline 65 & BY 19 & $5.27 \pm 0.49$ & $4.37 \pm 0.03$ & Zhangjiakou, Hebei, China \\
\hline 66 & BY 20 & $5.22 \pm 0.28$ & $4.03 \pm 0.02$ & Zhangjiakou, Hebei, China \\
\hline 67 & BY 21 & $5.55 \pm 0.42$ & $4.12 \pm 0.01$ & Zhangjiakou, Hebei, China \\
\hline 68 & BY 22 & $4.77 \pm 0.62$ & $4.07 \pm 0.13$ & Zhangjiakou, Hebei, China \\
\hline 69 & BY 23 & $5.31 \pm 0.58$ & $4.00 \pm 0.05$ & Zhangjiakou, Hebei, China \\
\hline 70 & BY 24 & $5.57 \pm 0.42$ & $4.13 \pm 0.04$ & Zhangjiakou, Hebei, China \\
\hline 71 & BY 25 & $4.80 \pm 0.19$ & $5.02 \pm 0.12$ & Zhangjiakou, Hebei, China \\
\hline 72 & BY 26 & $4.59 \pm 0.25$ & $4.61 \pm 0.08$ & Zhangjiakou, Hebei, China \\
\hline 73 & BY 27 & $5.76 \pm 0.71$ & $4.57 \pm 0.06$ & Zhangjiakou, Hebei, China \\
\hline 74 & BY 28 & $5.09 \pm 0.55$ & $4.37 \pm 0.00$ & Zhangjiakou, Hebei, China \\
\hline 75 & BY 29 & $5.00 \pm 0.50$ & $4.54 \pm 0.00$ & Zhangjiakou, Hebei, China \\
\hline 76 & BY 30 & $5.70 \pm 0.47$ & $3.43 \pm 0.02$ & Zhangjiakou, Hebei, China \\
\hline 77 & BY 31 & $5.41 \pm 0.83$ & $4.37 \pm 0.02$ & Zhangjiakou, Hebei, China \\
\hline 78 & BY 32 & $5.08 \pm 1.07$ & $4.04 \pm 0.07$ & Zhangjiakou, Hebei, China \\
\hline 79 & BY 33 & $4.43 \pm 0.47$ & $4.06 \pm 0.12$ & Zhangjiakou, Hebei, China \\
\hline 80 & BY 34 & $4.43 \pm 0.27$ & $5.17 \pm 0.00$ & Zhangjiakou, Hebei, China \\
\hline 81 & BY 35 & $5.01 \pm 1.03$ & $4.84 \pm 0.00$ & Zhangjiakou, Hebei, China \\
\hline 82 & BY 36 & $5.44 \pm 0.10$ & $4.37 \pm 0.01$ & Zhangjiakou, Hebei, China \\
\hline 83 & BY 37 & $5.26 \pm 1.03$ & $3.12 \pm 0.00$ & Zhangjiakou, Hebei, China \\
\hline 84 & BY 38 & $5.27 \pm 0.39$ & $3.13 \pm 0.01$ & Zhangjiakou, Hebei, China \\
\hline 85 & BY 39 & $4.69 \pm 0.78$ & $4.93 \pm 0.01$ & Zhangjiakou, Hebei, China \\
\hline 86 & BY 40 & $4.24 \pm 0.37$ & $3.90 \pm 0.02$ & Zhangjiakou, Hebei, China \\
\hline 87 & BY 41 & $4.85 \pm 1.05$ & $4.28 \pm 0.02$ & Zhangjiakou, Hebei, China \\
\hline 88 & BY 42 & $4.53 \pm 1.31$ & $4.45 \pm 0.01$ & Zhangjiakou, Hebei, China \\
\hline 89 & BY 43 & $4.54 \pm 0.42$ & $3.74 \pm 0.04$ & Zhangjiakou, Hebei, China \\
\hline 90 & BY 44 & $4.21 \pm 0.11$ & $4.03 \pm 0.07$ & Zhangjiakou, Hebei, China \\
\hline 91 & BY 45 & $5.01 \pm 0.71$ & $4.99 \pm 0.08$ & Zhangjiakou, Hebei, China \\
\hline 92 & BY 46 & $5.52 \pm 0.66$ & $4.16 \pm 0.07$ & Zhangjiakou, Hebei, China \\
\hline 93 & BY 47 & $5.64 \pm 0.49$ & $4.29 \pm 0.06$ & Zhangjiakou, Hebei, China \\
\hline
\end{tabular}


Table 2. Cont.

\begin{tabular}{ccccc}
\hline S. No. & Sample Name & Moisture Content (\%) & $\begin{array}{c}\beta \text {-Glucan } \% \text { w/w (Dry } \\
\text { wt. Basis) }\end{array}$ & Origin \\
\hline 94 & BY 48 & $5.11 \pm 0.42$ & $4.25 \pm 0.00$ & Zhangjiakou, Hebei, China \\
\hline 95 & BY 49 & $4.22 \pm 0.74$ & $3.45 \pm 0.04$ & Zhangjiakou, Hebei, China \\
\hline 96 & BY 50 & $4.54 \pm 0.16$ & $3.73 \pm 0.00$ & Zhangjiakou, Hebei, China \\
\hline 97 & BY 51 & $4.92 \pm 0.35$ & $4.43 \pm 0.00$ & Zhangjiakou, Hebei, China \\
\hline 98 & BY 52 & $4.69 \pm 0.79$ & $4.13 \pm 0.01$ & Zhangjiakou, Hebei, China \\
\hline 99 & BY 53 & $5.02 \pm 0.52$ & $3.99 \pm 0.02$ & Zhangjiakou, Hebei, China \\
\hline 100 & ZNY282 & $4.17 \pm 0.05$ & $4.45 \pm 0.05$ & Zhangjiakou, Hebei, China \\
\hline
\end{tabular}

Data were expressed as mean \pm standard deviation $(n=3), w / w$; weight/weight.

Figure 1 presents the NIR spectra of the hulled and naked oat samples. The absorption bands of both hulled and naked oat samples exhibit the least difference. The NIR spectra of samples were complex and present the prominent absorption bands around $1200 \mathrm{~nm}$, $1450 \mathrm{~nm}, 1750 \mathrm{~nm}, 1950 \mathrm{~nm}, 2100 \mathrm{~nm}, 2300 \mathrm{~nm}$, and $2500 \mathrm{~nm}$, whereas the lowest absorbance was observed in wavelength band 900-1091 nm.
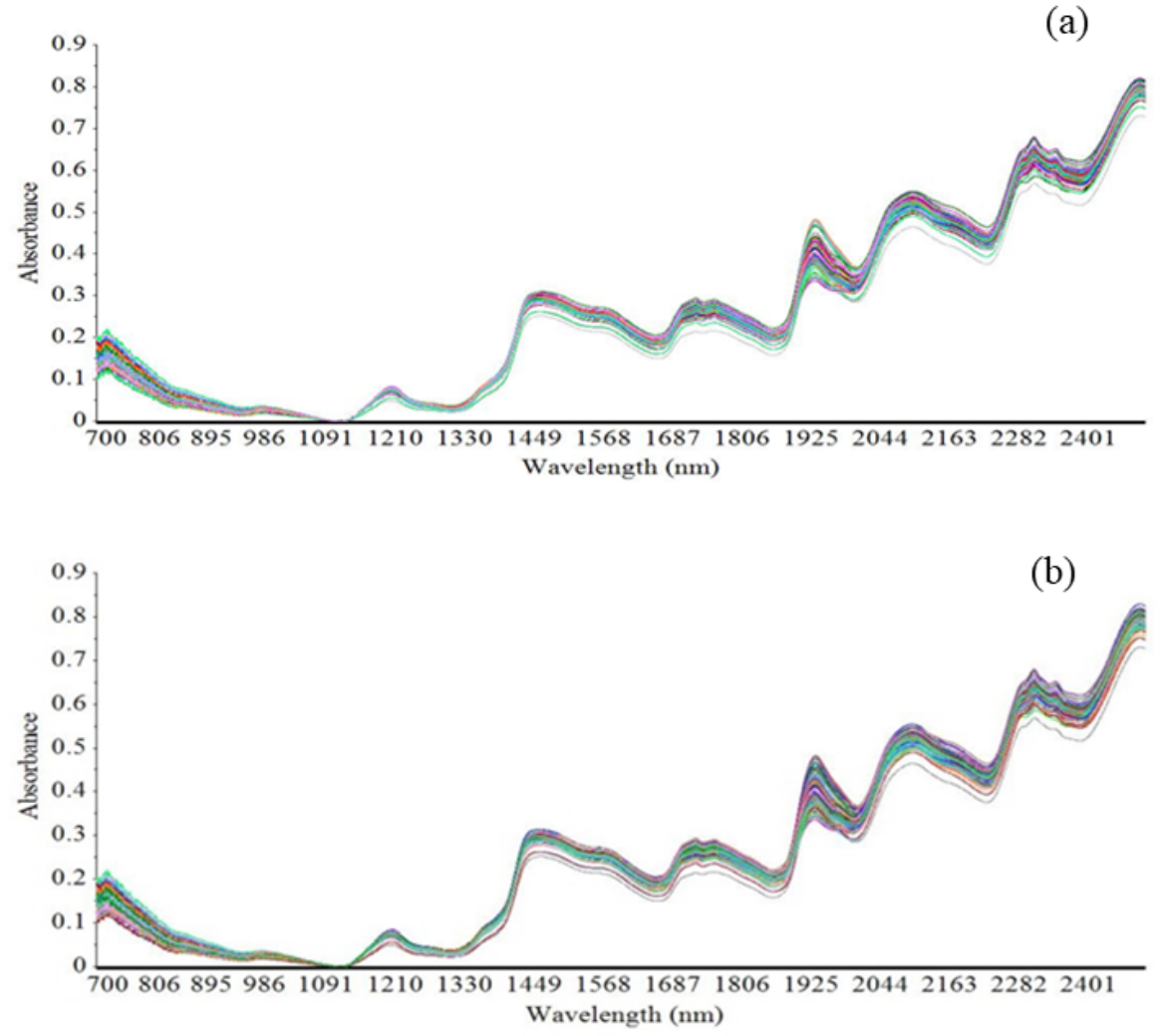

Figure 1. Typical near-infrared spectra of (a) hulled oat samples and (b) naked oat samples.

Thus, iPLS was performed for the selection of important wavelengths for the prediction of $\beta$-glucan in oats. Furthermore, the correlation matrix plots were employed to determine the correlation between each wavelength range and the $\beta$-glucan content. The correlation between wavelength range $700-1300 \mathrm{~nm}$ and 1400 to $1700 \mathrm{~nm}$ with the $\beta$-glucan content of hulled oat samples are shown in Figure 2a or Figure 2b, respectively. It can be observed that the wavelengths ranging from 1091 to $1218 \mathrm{~nm}$ present low correlation values (less than 0.2 ) compared with the other wavelengths (equal to greater than 0.2 ). The wavelengths from 1400 to $1700 \mathrm{~nm}$ also exhibit negligible correlation with $\beta$-glucan measurements. Thus, these 
wavelengths with less correlation were least important and considered as outliers for the determination of $\beta$-glucan to reduce the error, whereas the wavelength range 1300-1400 nm presented a strong negative correlation and the wavelength range 1700-1900 nm exhibits a strong positive correlation with $\beta$-glucan content. Thus, these wavelength ranges in group II with strong correlation were important for the prediction of $\beta$-glucan. Similarly, the important wavelengths (1950-2050 and 2150-2500 nm) were identified from group III (1900-2500 nm) using the correlation matrix, as shown in Figure 2c. The correlation matrix plots between $\beta$-glucan and the different wavelength ranges for the naked samples are presented in Figure $2 \mathrm{~d}-\mathrm{f}$. As shown in Figure 2e or Figure 2f, wavelength ranges 1850-1865 $\mathrm{nm}$ and 1900-2000 nm exhibit a negative correlation with $\beta$-glucan, respectively. The wavelengths with correlation values of more than 0.1 were used for the development of a model, and the other wavelengths with low correlation values were considered outliers for the development of regression model.
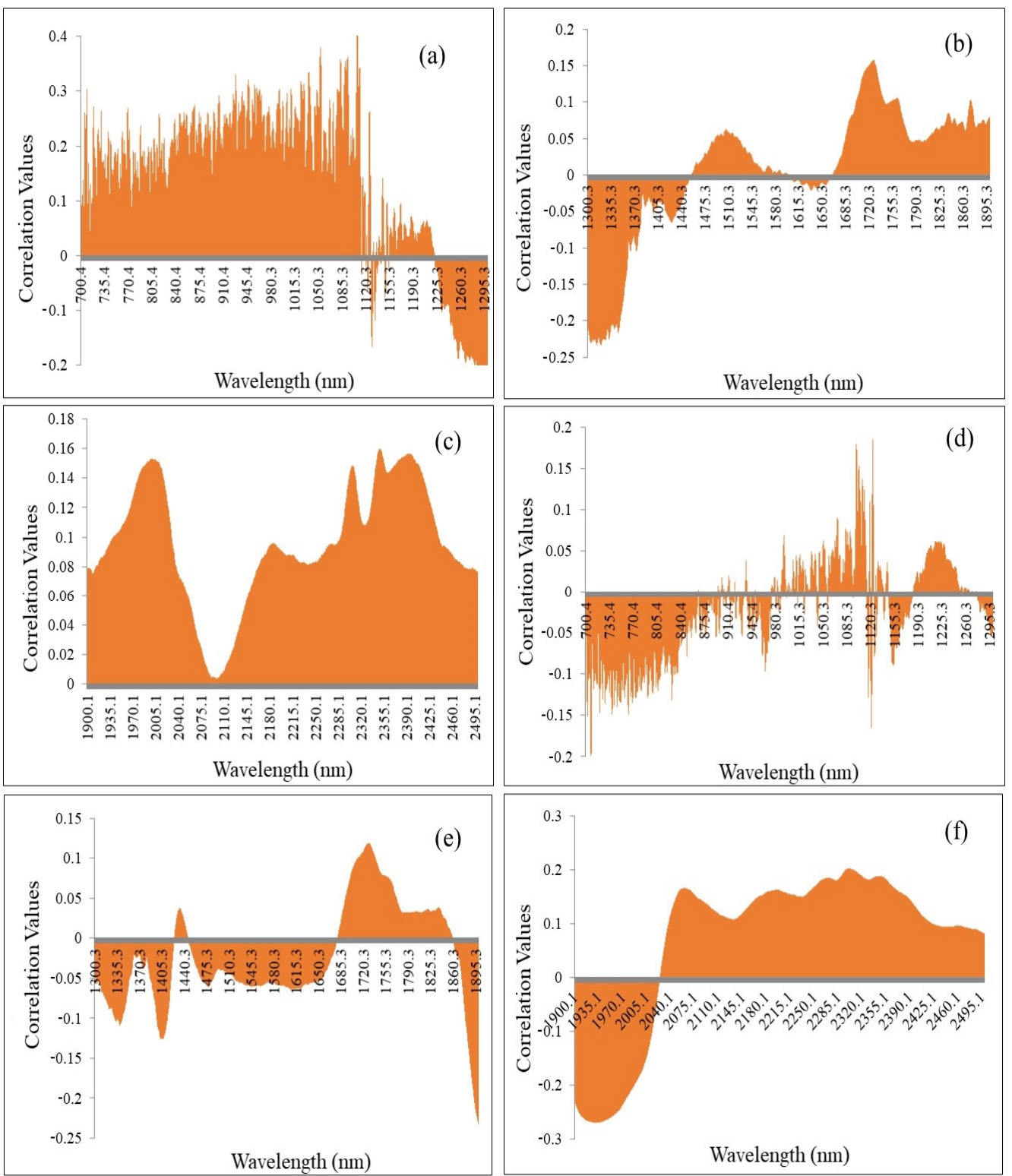

Figure 2. The correlation matrix plots of $\beta$-glucan content and NIR spectra in the wavelength ranges (a) 700-1300 nm, (b) 1300-1900 nm, and (c) 1900-2500 nm of hulled oats and (d) 700-1300 nm, (e) 1300-1900 nm, and (f) 1900-2500 nm of naked oats. 
The PLSR analysis was employed to determine the relationship between spectral absorbance and concentration of $\beta$-glucan by employing wavelengths extracted from iPLS. The Savitzky-Golay smoothening and normalization were applied to the spectral data before prediction analysis for reducing errors and for smoothening the spectral data. PLS loading plots for the hulled and naked samples are shown in Figure 3a-f, respectively. The loading plot shows the correlation loading coefficients for the regression equation developed to predict the $\beta$-glucan content in the oat samples. Each graph is divided into three parts: the upper part for the correlation loading value of $0.7-1$ represents the wavelengths with a strong positive correlation coefficient with $\beta$-glucan content, the middle part shows wavelengths with moderate or small correlation coefficients, and the lower part shows strong negative correlation coefficients. Wavelengths with strong positive and negative correlations were selected for the prediction model, and the PLSR analysis was applied for the three groups separately.

The correlation loading plot for the naked samples (Figure $3 \mathrm{~d}-\mathrm{f}$ ) showed that the wavelengths from 700-972 $\mathrm{nm}$ and 2100-2500 nm exhibit a strong positive correlation with the $\beta$-glucan of oats, whereas the wavelengths from $900-1300 \mathrm{~nm}$ present strong negative correlation coefficients with the $\beta$-glucan of naked oats. In the case of hulled oat samples, the correlation loading plots showed that the wavelength range from 700-900 nm exhibits a strong positive correlation and that the wavelength ranges 900-1900 and 2000-2500 nm present strong negative correlations (Figure 3a-c). Furthermore, it was also observed that the wavelength range 1900-2000 nm exhibits minimal to no correlation with $\beta$-glucan of hulled oat samples.

The prediction results for the $\beta$-glucan content of hulled and naked oats samples are shown in Figure 4 and Table 3 . The $R^{2} c$ and $R^{2} p$ for hulled oats was higher, and the root mean square error (RMSE) was also considerably lower in the case of the first interval ranging from 700 to $1300 \mathrm{~nm}$. A similar trend was also observed in the case of the naked samples. It was also observed that the $\mathrm{R}^{2} \mathrm{c}$ and $\mathrm{R}^{2} \mathrm{p}$ was slightly higher, and RMSE was comparatively lower in the case of hulled samples compared with naked samples in each wavelength interval. Overall, PLSR analysis in wavelength range 700-1300 nm can be successfully employed for the prediction of $\beta$-glucan content of hulled oats with $\mathrm{R}^{2} \mathrm{c}=0.789$, $\mathrm{R}^{2} \mathrm{p}=0.735, \mathrm{RMSEC}=0.177$, and $\mathrm{RMSEP}=0.199$, whereas in the case of naked oat samples, the best prediction results were also observed in the wavelength range 700-1300 nm with $\mathrm{R}^{2} \mathrm{c}=0.677, \mathrm{R}^{2} \mathrm{p}=0.620$, and $\mathrm{RMSEC}=0.210, \mathrm{RMSEP}=0.228$, but the $\mathrm{R}^{2} \mathrm{c}$ and $\mathrm{R}^{2} \mathrm{p}$ values were found to be poor in the second and third intervals ranging from 1300-1900 and $1900-2500 \mathrm{~nm}$ in both naked and hulled oats.

Table 3. Statistical results of partial least square regression models developed to predict the $\beta$-glucan content of hulled and naked oat samples.

\begin{tabular}{ccccccccc}
\hline \multirow{2}{*}{$\begin{array}{l}\text { Wavelength } \\
\text { Range (nm) }\end{array}$} & \multicolumn{4}{c}{ Hulled Oats } & \multicolumn{4}{c}{ Naked Oats } \\
\cline { 2 - 9 } & $\mathbf{R}^{\mathbf{2}} \mathbf{c}$ & $\mathbf{R}^{\mathbf{2}} \mathbf{p}$ & $\mathbf{R M S E C}$ & $\mathbf{R M S E P}$ & $\mathbf{R}^{\mathbf{2}} \mathbf{c}$ & $\mathbf{R}^{\mathbf{2}} \mathbf{p}$ & $\mathbf{R M S E C}$ & $\mathbf{R M S E P}$ \\
\hline $700-1300$ & 0.789 & 0.735 & 0.177 & 0.199 & 0.677 & 0.620 & 0.210 & 0.228 \\
\hline $1300-1900$ & 0.431 & 0.301 & 0.291 & 0.323 & 0.325 & 0.226 & 0.304 & 0.325 \\
\hline $1900-2500$ & 0.460 & 0.336 & 0.284 & 0.315 & 0.382 & 0.274 & 0.291 & 0.315 \\
\hline
\end{tabular}



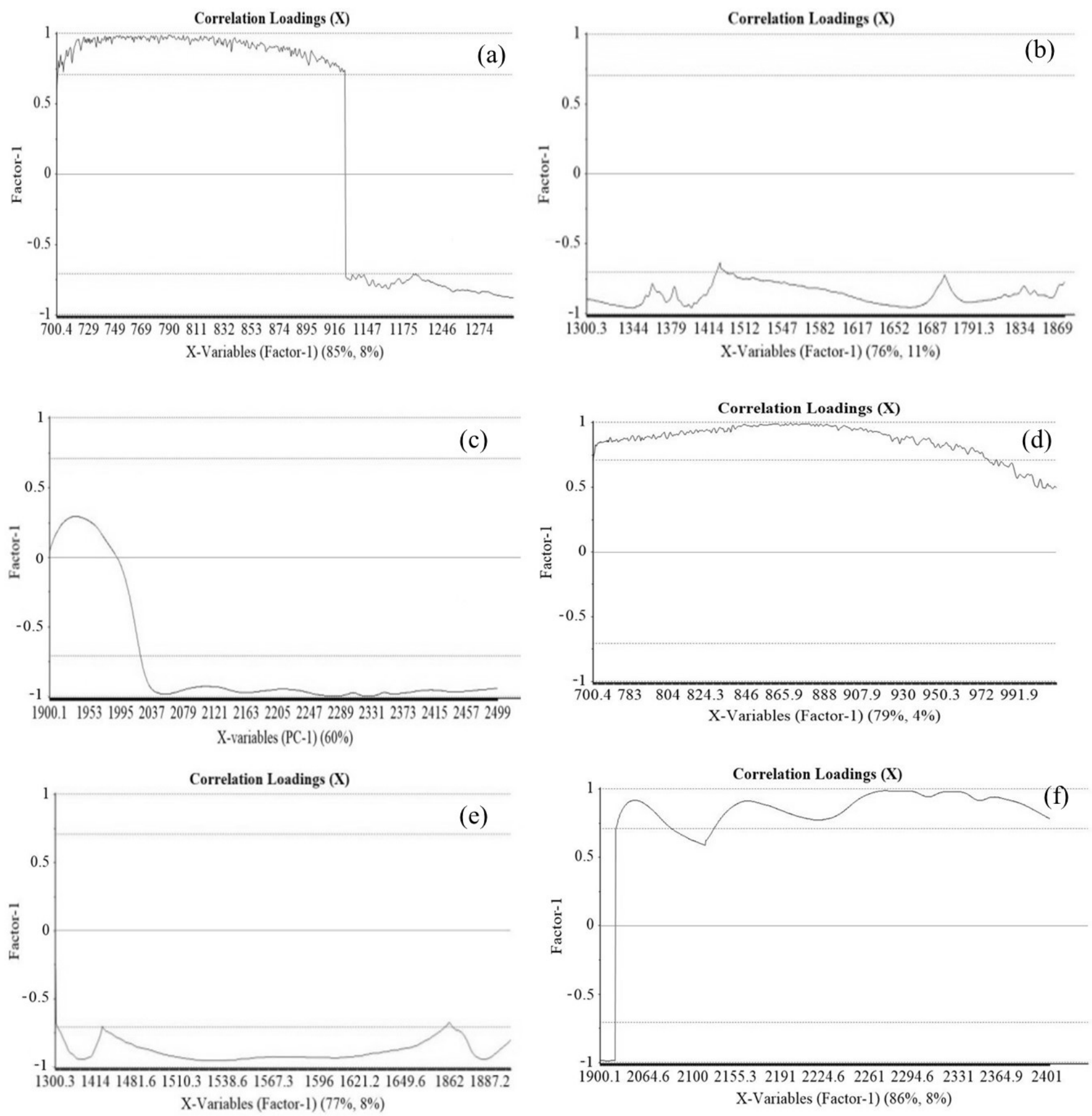

Figure 3. PLS loading plots in wavelength ranges (a) $700-1300 \mathrm{~nm}$, (b) 1300-1900 nm, and (c) 1900-2500 nm for hulled oats and (d) 700-1300 nm, (e) 1300-1900 nm, and (f) 1900-2500 nm for naked oats. 

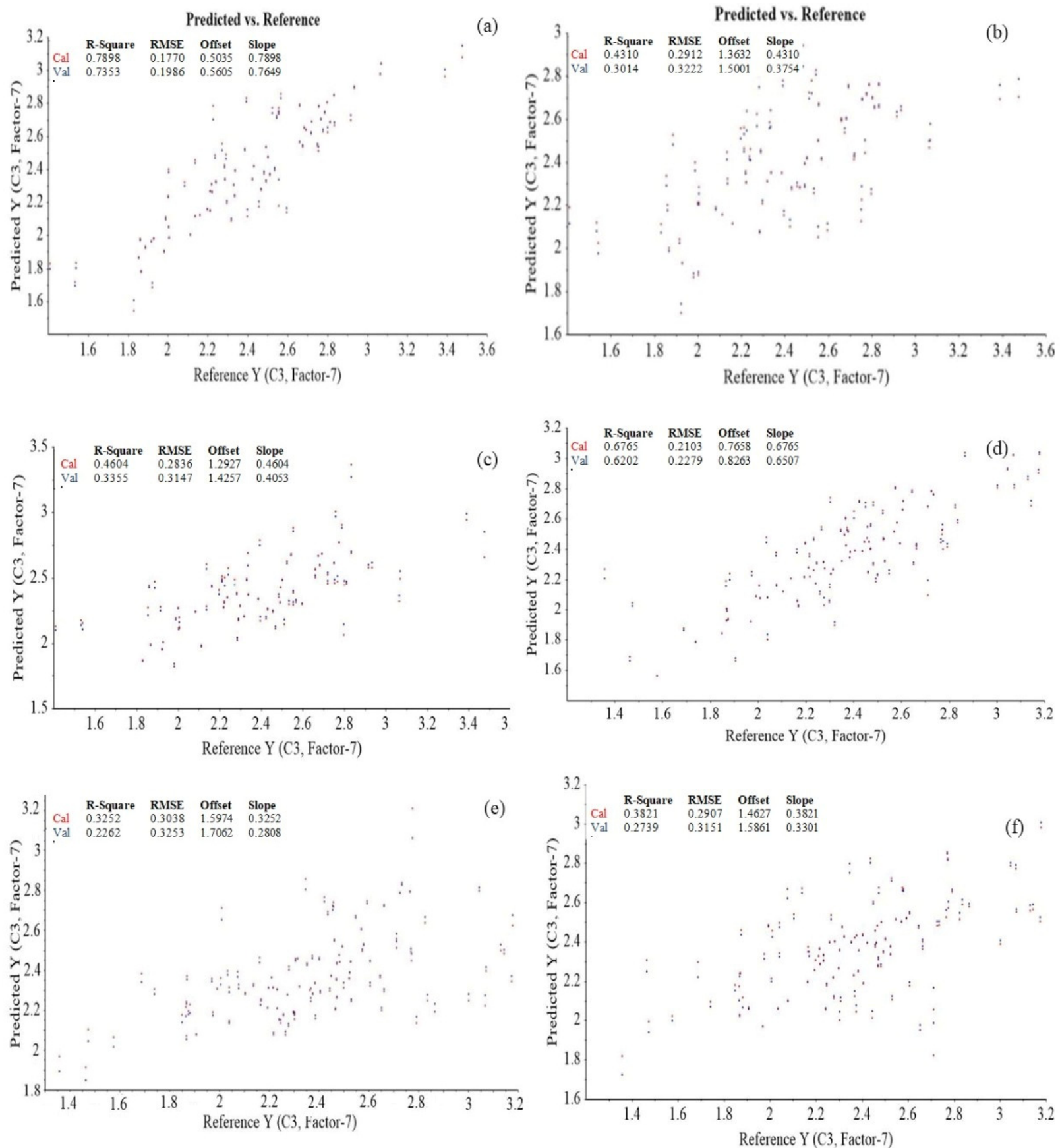

Figure 4. Partial least square model of $\beta$-glucan content in the wavelength ranges (a) 700-1300 nm, (b) 1300-1900 nm, and (c) 1900-2500 nm for hulled oats and (d) 700-1300 nm, (e) 1300-1900 nm, and (f) $1900-2500 \mathrm{~nm}$ for naked oats.

\section{Discussion}

The growing interest in functional foods accelerates research on plant materials rich in $\beta$-glucan. The health benefits of $\beta$-glucan such as antitumor, antioxidant, immunomodulatory, hypocholesterolemic, hypoglycemic, and anti-inflammatory activities are widely reported in the literature [3,5]. Thus, exploration of rich sources of $\beta$-glucan will lead to the development of novel plant material and food products with a high amount of $\beta$-glucan that, in turn, will provide various health benefits to consumers. In the present study, the $\beta$-glucan content of 100 different varieties of hulled oats and 79 varieties of naked oats were explored by employing the standard McCleary method. In case of hulled oat samples, the highest amount of $\beta$-glucan (5.5\%) was observed ZNY 258 from Zhangjiakou, Hebei, China, 
and the lowest amount (3.1\%) was found in the case of variety Zhangyan \#7, also collected from Zhangjiakou, Hebei, China, whereas in the case of naked oat samples, the highest amount $(5.22 \%)$ was observed in the case of variety BY 4 and the lowest amount (3.12\%) was found in the case of sample BY 37. Both of these oat varieties were also collected from Zhangjiakou, Hebei, China. The $\beta$-glucan content of oat samples observed in the present study coincides well with the findings of previous studies $[4,8]$. In addition, the coefficient of variation among the $\beta$-glucan values of hulled and naked oat samples was found to be $10.67 \%$ and $10.79 \%$. Thus, the chemometrics model established based on the spectral and this reference data will be able to predict the wide range of $\beta$-glucan in different oats varieties. A similar approach was also applied in a previous study while developing prediction models for phenolic compounds based on the NIR spectroscopy [10].

The NIR spectra of hulled and naked oats were collected from 700 to $2500 \mathrm{~nm}$. The NIR spectra of oat samples were complex due to the presence of overtone vibrations and a combination of bands of $\mathrm{C}-\mathrm{H}$ (aliphatic), $\mathrm{C}-\mathrm{H}$ (aromatic), $\mathrm{C}-\mathrm{O}$ (carboxyl), O-H (hydroxyl), and $\mathrm{N}-\mathrm{H}$ (amine and amide) bonds present in organic compounds present in the oat samples [10,14,21]. The major absorption bands observed around $1200 \mathrm{~nm}$ correspond to the $\mathrm{C}-\mathrm{H}$ 2nd overtone; $1450 \mathrm{~nm}$ corresponds to the $\mathrm{O}-\mathrm{H}$ 1st overtone and the $-\mathrm{CH}_{2}$, $-\mathrm{CH}_{3}$, and $-\mathrm{CH}=\mathrm{CH}-1$ st overtone; $1750 \mathrm{~nm}$ corresponds to the $\mathrm{C}=\mathrm{O}$ stretch 2 nd overtone; $1950 \mathrm{~nm}$ corresponds to a combination of the stretching mode and the deformation mode of $\mathrm{H} 2 \mathrm{O} ; 2100 \mathrm{~nm}$ presents a combination of the $\mathrm{O}-\mathrm{H}$ deformation mode and the $\mathrm{C}-\mathrm{O}$ stretching mode; $2300 \mathrm{~nm}$ presents the $-\mathrm{CH}_{3}$ combination; and $2500 \mathrm{~nm}$ corresponds to a combination of $\mathrm{C}-\mathrm{H}$ and $\mathrm{C}-\mathrm{C}$ stretching $[10,13,22]$. The lowest absorbance was observed in the wavelength band 900-1091 nm. Similar absorption bands were also reported previously in the case of NIR spectra of oat groats [13]. Thus, due to the complex nature of the NIR spectra of oat samples, a multivariate statistical analysis needs to be conducted using chemical data as primary data and spectral data as secondary data for a better interpretation of the spectra, for the extraction of useful information, and for a quantitative analysis of the chemical components present in a plant material [23].

It was also mentioned that the wavelengths corresponding to low absorbance bands may be considered unwanted as these wavelengths contribute towards error during the establishment of a statistical model for the prediction of $\beta$-glucan in oats [22]. Thus, to extract important wavelengths for the prediction of $\beta$-glucan, iPLS was performed. In iPLS, the whole spectral data were subdivided into non-overlapping equal sections that undergo PLSR modelling to retrieve the most useful wavelength range (with high correlation coefficient and low RMSE) for the prediction of a particular biochemical parameter [24]. The resultant correlation matrix plots present the relationship between a particular biochemical parameter and absorption at different wavelengths. The wavelengths with less correlation values are mentioned as least important for the prediction of a biochemical parameter under investigation and can be rejected because they describe noise or other minor components [25]. In present study, the complete wavelength range (700-2500 nm) was divided into three equal parts (700-1300, 1300-1900 and 1900-2500 nm), and iPLS was performed. The resultant correlation matrix plots in Figure 2a revealed the shift in correlation values from high positive correlation to high negative correlation with an increase in the wavelength. The wavelength range from 700-1100 nm showed a positive but inconsistent correlation, 1120-1220 nm exhibited no or minimal correlation, and wavelength range 1225-1300 nm presented a high negative correlation with the $\beta$-glucan content of hulled oats. Thus, the wavelengths from 1120 to $1220 \mathrm{~nm}$ were not included in the development of the PLSR model for the prediction of $\beta$-glucan content. Furthermore, a negative correlation was observed between $\beta$-glucan content and wavelength range $1300-1440 \mathrm{~nm}$ (Figure 2b), whereas the highest wavelength ranges, 2040-2140 nm, showed a positive and feeble correlation (Figure 2c) and thus was not employed for the establishment of the PLSR models. However, the wavelength ranges 1950-2050 and 2150-2500 exhibited a satisfactory positive correlation with $\beta$-glucan content. Thus, they were employed for PLSR model development for the prediction of $\beta$-glucan content of hulled oats. In addition, 
the correlation matrix plots for different wavelength ranges (700-1300, 1300-1900, and $1900-2500 \mathrm{~nm}$ ) and $\beta$-glucan contents of naked oats are presented in Figure $2 \mathrm{~d}-\mathrm{f}$. The initial wavelengths till around $975 \mathrm{~nm}$ (Figure 2d) were negative, followed by an inconsistent and slightly positive correlation from 980 to $1150 \mathrm{~nm}$ and slightly positive correlation from around 1190 to $1260 \mathrm{~nm}$ followed by a negative correlation from 1290 to $1420 \mathrm{~nm}$ and 1442 to $1685 \mathrm{~nm}$ and a slightly positive correlation between $\beta$-glucan content and wavelength range 1686 to $1860 \mathrm{~nm}$ for naked oat samples (Figure 2e), whereas wavelength range 1900-2000 nm exhibits a negative correlation and wavelength range 2040 to $2500 \mathrm{~nm}$ exhibits a positive correlation with $\beta$-glucan of naked oats, as shown in Figure $2 \mathrm{f}$. The wavelengths with correlation values more than and equal to 0.2 were employed for the establishment of a model, whereas other wavelengths with low correlation values were considered outliers for the development of the regression model. A similar approach was also employed for the selection of important wavelengths for the development of efficient calibration and prediction models for phenolic compounds of mung bean [10].

Furthermore, a PLSR analysis was performed to elucidate the relationship between $\beta$-glucan of oat samples and spectral absorbance using wavelengths extracted from iPLS. Before applying PLSR analysis, the spectral data were pretreated by employing SavitzkyGolay smoothening and normalization techniques to effectively remove scattering effects and noises such as tilt, baseline-drift, and reverse [26,27]. PLSR was applied to the data by the NIPALS algorithm and cross-validation technique [28]. In the case of 79 hulled samples, 63 were used to develop the model and 16 samples were employed for validation of the model. However, among the 100 naked samples, 80 samples were employed for calibration and 20 were used for validation of the model. The data was processed using seven principal components. It was observed that $94 \%$ of the complete data can be employed for the regression using two orthogonal principal components. Furthermore, the line loading plots of the PLS wavelength ranges (a) 700-1300 nm, (b) 1300-1900 nm, and (c) 1900-2500 nm for hulled and naked oats were also explored to support the finding of correlation matrix plots. The results of loading plots well supported the results of correlation matrix plots. In the PLS loading plots, the wavelengths with correlation values in the range of 0.7 to 1 were regarded as important for the model development and prediction of $\beta$-glucan content compared with the wavelength with values from -0.7 to 0 to +0.7 . It was also observed that the initial wavelengths in the range of 700 to $1300 \mathrm{~nm}$ exhibited a positive correlation above or equal to 0.7 (Figure 3a), whereas the wavelength range 1300 to $1900 \mathrm{~nm}$ exhibited a negative correlation value (Figure 3b). In addition, the wavelengths in range 1900-2500 nm were also found to be least important for the prediction of $\beta$-glucan content, as their correlation values were below 0.7 to 1 (Figure $3 c$ ). In the case of naked oat samples, the wavelength range 700-900 nm was found to be important for the prediction of $\beta$-glucan content as their correlation value was between 0.7 to 1 (Figure $3 \mathrm{~d}$ ), whereas wavelengths from 1300 to $1900 \mathrm{~nm}$ exhibited negative correlation values below -0.5 and are thus regarded as outliers (Figure 3e) and the wavelength range 2000-2500 nm exhibited a variable but positive correlation between 0.7 to 1 (Figure 3f). Thus, it may also be considered important for the prediction of $\beta$-glucan content. Overall, in the case of Figure $3 \mathrm{a}-\mathrm{c}$, the wavelengths from 700-916 nm exhibited the highest positive correlation coefficient, whereas the wavelength region 1300-1900 nm (Figure 3) exhibited strong negative correlation coefficients. The wavelengths with high positive and negative correlations have equal importance in the prediction of $\beta$-glucan contents. The wavelength region 2000-2500 nm also presented a negative correlation coefficient; thus, wavelengths higher than $2100 \mathrm{~nm}$ are important for $\beta$-glucan prediction. In the case of both hulled and naked oat samples, the wavelengths less than $900 \mathrm{~nm}$ exhibited a strong positive correlation value. The wavelengths showing high correlation values for $\beta$-glucan content are more important for its prediction in the samples. Thus, the wavelengths showing a strong correlation with $\beta$-glucan from Figures 2 and 3 were chosen for the final PLSR model development.

Table 3 and Figure 4 present the results of the prediction results of $\beta$-glucan from the PLSR models. In each wavelength interval, the wavelengths presenting a high correlation 
with $\beta$-glucan content were employed for establishing the PLSR model for the oat samples. In contrast, the wavelengths with minimal or no correlation were considered outliers and not employed for the establishment of calibration models. It can be observed from Table 3 that the PLS prediction model results are in accordance with the results obtained from correlation loading and correlation matrix results. The wavelength interval from 700 to $1300 \mathrm{~nm}$ exhibits relatively good $\mathrm{R}^{2}$ values for calibration and prediction compared with the models developed from 1300-2500 nm in both hulled and naked oats. The high values for $R^{2} c$ and $R^{2} p$ and lower values for RMSE present the good performance of the calibration and prediction model [29-31]. In wavelength range $1300-1900 \mathrm{~nm}$, the $R^{2} \mathrm{c}$ and $\mathrm{R}^{2} \mathrm{p}$ values were poor for both hulled (0.431 and 0.301) and naked oat samples (0.325 and 0.226). Similar results were also observed in wavelength range $1900-2500 \mathrm{~nm}$ with $R^{2} \mathrm{c}$ and $R^{2} \mathrm{p}$ of 0.460 and 0.336 for hulled and 0.382 and 0.274 for naked oats, respectively, whereas in the spectral range from 700 to $1300 \mathrm{~nm}$, the $R^{2} \mathrm{c}$ and $\mathrm{R}^{2} \mathrm{p}$ for naked oats were 0.677 and 0.620 , and the highest values for $R^{2} c(0.789)$ and $R^{2} p(0.735)$ and the minimum values for RMSEC (0.177) and RMSEP (0.199) were observed for the hulled oat samples. Previously, it was mentioned that the calibration models with $R^{2} c$ between 0.66 and 0.81 present satisfactory predictions whereas prediction of chemical properties with $R^{2} c<0.5$ are reported as being unreliable [32-34]. A study by Seefeldt et al. also reported the best correlation of $\beta$-glucan of barley with the wavelengths in these regions [18]. Thus, in the future, the spectral region ranging from 700 to $1300 \mathrm{~nm}$ can be efficiently employed for the prediction of $\beta$-glucan of hulled oats to save time, whereas this wavelength range can also be employed for the satisfactory prediction of $\beta$-glucan of naked oats. In addition, a rapid and low-cost NIR spectrometer can also be designed by employing filters corresponding to $700-1300 \mathrm{~nm}$ for this particular application.

\section{Conclusions}

The rapid and non-destructive prediction of $\beta$-glucan content of hulled and naked oats was carried out by employing chemical data of the $\beta$-glucan content of oats (primary data) and NIR spectral data of oats (secondary data). The important wavelengths for the prediction of $\beta$-glucan of oats were selected using iPLS. The regression analysis was run on parts of the spectral data to check the range of spectra showing high coefficients of correlation with low error, chosen to build the best regression model for the prediction of the $\beta$-glucan content. The iPLS prediction and correlation matrix plots revealed the importance of wavelength range $700-1300 \mathrm{~nm}$ for the accurate prediction of $\beta$-glucan contents in hulled and naked oats samples. Various pre-processing techniques such as Savitzky-Golay smoothening and normalization were applied before prediction analysis. Applied pre-processing techniques helped in smoothening the data, which further helped in the analysis to understand the complex spectra. The results obtained from PLSR analysis indicate that the chemical data of $\beta$-glucan in hulled and naked oats along with the NIR spectra can be used for an accurate prediction of the $\beta$-glucan content with $\mathrm{R}^{2} \mathrm{c}$ of 0.789 and 0.677 for hulled and naked samples, respectively, in the wavelength range of 700-1300 nm with RMSEC and RMSEP less than 0.229 .

Author Contributions: Conceptualization, B.X. and P.H.; methodology, M.M. and Y.Z.; software, U.K. and P.H.; validation, B.X., P.H. and S.Z.; formal analysis, M.M., Y.Z. and U.K.; investigation, M.M. and Y.Z.; resources, S.Z. and L.C.; data curation, U.K. and P.H.; writing-original draft preparation, M.M. and Y.Z.; writing-review and editing, B.X.; visualization, B.X. and P.H.; supervision, B.X.; project administration, B.X. and S.Z.; funding acquisition, B.X. All authors have read and agreed to the published version of the manuscript.

Funding: This research was jointly funded by two grants R202007 and R202017 from BNU-HKBU United International College, China.

Data Availability Statement: Data are available upon request. 
Acknowledgments: Authors are thankful to CSIR-CSIO, Chandigarh (India), for providing access to "The Unscrambler" software.

Conflicts of Interest: The authors declare no conflict of interest.

\section{References}

1. FAO. FAOSTAT, Food and Agriculture Organization of the United Nations (FAO). Available online: http://www.fao.org/faostat/ en/\#data/QC (accessed on 3 April 2020).

2. Suttie, J.M.; Reynolds, S.G. Fodder Oats: A World Overview; Food and Agriculture Organization of the United Nations: Rome, Italy, 2004.

3. Yoo, H.U.; Ko, M.J.; Chung, M.S. Hydrolysis of beta-glucan in oat flour during subcritical-water extraction. Food Chem. 2020, 308, 125670. [CrossRef]

4. Redaelli, R.; Del Frate, V.; Bellato, S.; Terracciano, G.; Ciccoritti, R.; Germeier, C.U.; De Stefanis, E.; Sgrulletta, D. Genetic and environmental variability in total and soluble $\beta$-glucan in European oat genotypes. J. Cereal Sci. 2013, 57, 193-199. [CrossRef]

5. Du, B.; Meenu, M.; Liu, H.; Xu, B. A concise review on the molecular structure and function relationship of $\beta$-glucan. Int. J. Mol. Sci. 2019, 20, 4032. [CrossRef]

6. Du, B.; Meenu, M.; Xu, B. Insights into improvement of physiochemical and biological properties of dietary fibers from different sources via micron technology. Food Rev. Int. 2020, 36, 367-383. [CrossRef]

7. Du, B.; Bian, Z.; Xu, B. Skin health promotion effects of natural beta-glucan derived from cereals and microorganisms: A review. Phyther. Res. 2014, 28, 159-166. [CrossRef] [PubMed]

8. Dotsenko, G.; Andersson, A.A.M.; Andersson, R. Material disintegration affects enzymatic determination of $\beta$-glucan in barley and oats. J. Cereal Sci. 2019, 88, 138-144. [CrossRef]

9. McCleary, B.V.; Codd, R. Measurement of $(1 \rightarrow 3),(1 \rightarrow 4)-\beta$-D-glucan in barley and oats: A streamlined enzymic procedure. J. Sci. Food Agric. 1991, 55, 303-312. [CrossRef]

10. Meenu, M.; Kamboj, U.; Sharma, A.; Guha, P.; Mishra, S. Green method for determination of phenolic compounds in mung bean (Vigna radiata L.) based on near-infrared spectroscopy and chemometrics. Int. J. Food Sci. Technol. 2016, 51, 2520-2527. [CrossRef]

11. Sakudo, A. Near-infrared spectroscopy for medical applications: Current status and future perspectives. Clin. Chim. Acta 2016, 455, 181-188. [CrossRef]

12. Deidda, R.; Sacre, P.; Clavaud, M.; Coïc, L.; Avohou, H.; Hubert, P.; Ziemons, E. Vibrational spectroscopy in analysis of pharmaceuticals: Critical review of innovative portable and handheld NIR and Raman spectrophotometers. Trends Anal. Chem. 2019, 114, 251-259. [CrossRef]

13. Redaelli, R.; Berardo, N. Evaluation of groat percentage in oats (Avena sativa L.) by near infrared spectroscopy. J. Near Infrared Spectrosc. 2002, 109, 103-109. [CrossRef]

14. Redaelli, R.; Berardo, N. Prediction of fibre components in oat hulls by near infrared reflectance spectroscopy. J. Sci. Food Agric. 2007, 585, 580-585. [CrossRef]

15. Kays, S.E.; Barton, F.E.; Windham, W.R. Predicting protein content by near infrared reflectance spectroscopy in diverse cereal food products. J. Near Infrared Spectrosc. 2000, 44, 35-43. [CrossRef]

16. Cristina, S.; Milach, K.; Agriscience, C. Near infrared reflectance spectroscopy (NIRS) to assess protein and lipid Near infrared reflectance spectroscopy (NIRS) to assess protein and lipid contents in Avena sativa L. Crop Breed. Appl. Biotechnol. 2008, 8, 127-133. [CrossRef]

17. Wang, N.; Zhang, X.; Yu, Z.; Li, G.; Zhou, B. Quantitative analysis of adulterations in oat flour by FT-NIR spectroscopy, incomplete unbalanced randomized block design, and partial least squares. J. Anal. Methods Chem. 2014, 2014, 393596. [CrossRef]

18. Seefeldt, H.F.; Blennow, A.; Jespersen, B.M.; Wollenweber, B.; Engelsen, S.B. Accumulation of mixed linkage (1/3) (1/4)-b-D-glucan during grain filling in barley: A vibrational spectroscopy study. J. Cereal Sci. 2009, 49, 24-31. [CrossRef]

19. Bellato, S.; Del Frate, V.; Redaelli, R.; Sgrulletta, D.; Bucci, R.; Magrì, A.D.; Marini, F. Use of near infrared reflectance and transmittance coupled to robust calibration for the evaluation of nutritional value in naked oats. J. Agric. Food Chem. 2011, 59, 4349-4360. [CrossRef] [PubMed]

20. Schmidt, J.; Gergely, S.; Schönlechner, R.; Grausgruber, H.; Tömösközi, S.; Salgó, A.; Berghofer, E. Comparison of different types of NIR instruments in ability to measure $\beta$-glucan content in naked barley. Cereal Chem. 2009, 86, 398-404. [CrossRef]

21. Gracia, M.B.; Armstrong, P.R.; Rongkui, H.; Mark, S. Quantification of betaglucans, lipid and protein contents in whole oat groats (Avena sativa L.) using near infrared reflectance spectroscopy. J. Near Infrared Spectrosc. 2017, 25, 172-179. [CrossRef]

22. Paudel, D.; Caffe-Treml, M.; Krishnan, P. A single analytical platform for the rapid and simultaneous measurement of protein, oil, and $\beta$-glucan contents of oats using near-infrared reflectance spectroscopy. Cereal Foods World 2018, 63, 17-25.

23. Biancolillo, A.; Marini, F. Chemometrics Applied to Plant Spectral Analysis. In Comprehensive Analytical Chemistry; Lopes, J., Sousa, C., Eds.; Elsevier: Amsterdam, The Netherlands, 2018; Volume 80, pp. 69-104. ISBN 9780444640482.

24. Nørgaard, L.; Saudland, A.; Wagner, J.; Nielsen, J.P.; Munck, L.; Engelsen, S.B. Interval Partial Least-Squares Regression (iPLS): A Comparative Chemometric Study with an Example from Near-Infrared Spectroscopy. Appl. Spectrosopy 2016, 54, 413-419. [CrossRef] 
25. Frost, T. Quantitative Analysis. In Encyclopedia of Spectroscopy and Spectrometry; Lindon, J.C., Ed.; Academic Press: Cambridge, MA, USA, 1999; pp. 2325-2330. ISBN 9780123744135.

26. Jiao, Y.; Li, Z.; Chen, X.; Fei, S. Preprocessing methods for near-infrared spectrum calibration. J. Chemom. 2020, 34 , e3306. [CrossRef]

27. Savitzky, A.; Golay, M.J.E. Smoothing and differentiation of data by simplified least squares procedures. Anal. Chem. 2002, 36, 1627-1639. [CrossRef]

28. Martens, H.; Næs, T. Multivariate Calibration; John Wiley \& Sons: New York, NY, USA, 1989; ISBN 0471909793.

29. Meenu, M.; Cai, Q.; Xu, B. A critical review on analytical techniques to detect adulteration of extra virgin olive oil. Trends Food Sci. Technol. 2019, 91, 391-408. [CrossRef]

30. Meenu, M.; Decker, E.A.; Xu, B. Application of vibrational spectroscopic techniques for determination of thermal degradation of frying oils and fats: A review. Crit. Rev. Food Sci. Nutr. 2021, 1891520. [CrossRef]

31. Meenu, M.; Xu, B. Application of vibrational spectroscopy for classification, authentication and quality analysis of mushroom: A concise review. Food Chem. 2019, 289, 545-557. [CrossRef]

32. Soriano-Disla, J.M.; Janik, L.J.; Rossel, R.A.V.; MacDonald, L.M.; McLaughlin, M.J. The performance of visible, near-, and mid-infrared reflectance spectroscopy for prediction of soil physical, chemical, and biological properties. Appl. Spectrosc. Rev. 2013, 49, 139-186. [CrossRef]

33. Recha, J.W.; Olale, K.O.; Sila, A.; Ambaw, G.; Radeny, M.; Solomon, D. Ensemble modeling on near-infrared spectra as rapid tool for assessment of soil health indicators for sustainable food production systems. Soil Syst. 2021, 5, 69. [CrossRef]

34. Rodríguez-Pérez, J.R.; Marcelo, V.; Pereira-Obaya, D.; García-Fernández, M.; Sanz-Ablanedo, E. Estimating soil properties and nutrients by visible and infrared diffuse reflectance spectroscopy to characterize vineyards. Agronomy 2021, 11, 1895. [CrossRef] 